\title{
0. cincis a \\ Interdisciplinary Research Drivers and Serendipity Factor: An Applied Mechanics Perspective on Some Past Application Platforms
}

\section{Chih-Kung Lee ${ }^{1}, 2,3$, *, Po-Cheng Lai ${ }^{1}$, Wen-Chi Chang1, Yi-Ching Kuo $^{2}$, Pei-I Tsai ${ }^{2}$, Ming-Han Chung ${ }^{1}$ and Julie T. Lee ${ }^{4}$}

${ }^{1}$ Institute of Applied Mechanics, National Taiwan University, Taipei, 10617, Taiwan

${ }^{2}$ Department of Engineering Science \& Ocean Engineering, National Taiwan University, Taipei, 10617, Taiwan

${ }^{3}$ Research Center for Applied Sciences, Academia Sinica, Nankang, Taipei, 11529, Taiwan

${ }^{4}$ AHEAD Optoelectronics, Inc. Chungho, New Taipei City, 23585 Taiwan

(Received 16 September 2014; Accepted 30 September 2014; Published on line 1 December 2014)

*E-mail: cklee@ntumems.net

DOI: $10.5875 /$ ausmt.v4i4.846

Abstract: In recent years, academia, research institutes, and industry have placed an increasing emphasis on interdisciplinary research, with a special focus on Applied Mechanics due to its influence on many emerging fields. Leaders in the Applied Mechanics field have emphasized the need to create an appropriate interdisciplinary organization, either through a matrix organization or with a formal organization. This paper reflects on the lessons learned from the inception, rise and evolution of the field of Theoretical \& Applied Mechanics at various universities and institutes around the world. In this way, we can obtain a better understanding of the driving forces of one type of interdisciplinary research. Collaboration and competition between the various disciplinary research institutes and the more traditional engineering/applied sciences departments will be discussed. Based on a series of research and development experiences and using Nano-BioMEMS/NEMS, smart structure, and electret application platforms, we discuss the theoretical architectures and corresponding applications to further strengthen our claim that successful interdisciplinary applied science research requires the establishment of strong industries. We also account for an added serendipity factor, which suggests that a prepared, well-trained open mind, connected to other collaborative research efforts, has the ability to seize new opportunities for innovation as they arise. Examples from past research provide suggestions on how to further improve interdisciplinary research. Other factors including the influence of an interdisciplinary organizational structure, collaborative teamwork framework, and the influence of online interaction with an exchange of ideas are also discussed.

Keywords: Interdisciplinary research; theoretical \& applied mechanics; Nano-BioMEMS/NEMS; smart structures; electrets; technology management

\section{Introduction}

It is generally acknowledged that modern higher education originated at Humboldt University of Berlin, founded in 1809 by Prussian educational reformer Wilhelm von Humboldt. He believed that a modern university should encompass "the sum of all knowledge", that teaching and research should be carried out in tandem, that students and researchers should enjoy 
academic freedom, and that the ultimate purpose of the university is to contribute to the completeness of knowledge [1]. Over the past century, considerable debate has emerged over the classification and differentiation of academic fields in higher education, which has led to the emergence of the concept and practice of interdisciplinary research. Boundaries between basic sciences, applied sciences, and interdisciplinary research are treated differently in different countries, leading to highly different outcomes. For example, twenty-nine members of the Cavendish Laboratory at the University of Cambridge's Physics Department have won Nobel Prizes in fields including physics, chemistry, and physiology and medicine [2]. With today's rapid industrial advancement and the establishment of different disciplines to meet the needs of new industries, critics have suggested that the education and research disciplines have become an assembly line which produces talent for industrial needs, rather than a mechanism for exploration and discovery that truly contributes to "the sum of all knowledge" [3]. Herein, we examine the nature of interdisciplinary research in the engineering fields of advanced industrial applications, and discuss the drivers of interdisciplinary research, including what we call an additional serendipity

Chih-Kung (C.K.) Lee received his Ph.D. from Cornell University. He worked at IBM's Almaden Research Center in San Jose, California before joining the faculty of National Taiwan University's Institute of Applied Mechanics in 1994 where he co-founded the National Taiwan University Nano-Bio-MEMS research group. He has served as Director-General of Engineering \& Applied Sciences, Taiwan's National Science Council (now the Ministry of Science and Technology), Executive Vice President of Taiwan's Industrial Technology Research Institute (ITRI), and President of the Institute for Information Industry (III). Since April 2013, he has been CEO and co-PI for Phase II of Taiwan's National Energy Program.

Po-Cheng Lai is a Ph.D. candidate at the Institute of Applied Mechanics, National Taiwan University. His research interests include piezoelectric sensors \& actuators, and acoustics.

Wen-Chi Chang is a Ph.D. candidate at the Institute of Applied Mechanics, National Taiwan University. Her research interests are on opto-fludics and electrets.

Yi-Ching Kuo is a Ph.D. candidate at the Department of Engineering Sciences \& Ocean Engineering, National Taiwan University. Her research interests include electrical impedance-based bio-metrology instruments and biochip design.

Pei-I Tsai is a Ph.D. candidate at the Department of Engineering Sciences \& Ocean Engineering, National Taiwan University. She was Deputy Director of Research Strategy Planning at Taiwan's Hsinchu Biomedical Science Park before serving at the Science and Technology Advisory Group of Taiwan's Executive Yuan. Her research interests include electrical impedance-based bio-metrology instruments and biochips.

Ming-Han Chung is a Ph.D. candidate at the Institute of Applied Mechanics, National Taiwan University. His research interests include nanowriter-based optical instruments/machining and light-field cameras.

Julie T. Lee received her B.A. from the University of Pennsylvania and her MBA from the University of Michigan. She has worked at AHEAD Optoelectronics, Inc. as International Manager since 1995. factor. We track the inception, rise and evolution of the field of Applied Mechanics as a point of initial reflection. In addition, since automation technologies can be viewed as a representative milestone in today's manufacturing industry, especially in Taiwan, the history of automation research will be used to examine possible directions to further advance this important interdisciplinary research and education field.

Since the 1980s, automation has played an increasingly important role in industrial production. In 1981, Taiwan's National Science Council (NSC) created an Automation Division within its organization [4]. In recent years, automation has played a role in promoting industrial progress by creating a high value-add paradigm shift based on smart automation using sensors, high-speed communication, intelligent algorithms and rules, analytics and "Big Data". Moreover, a new trend has emerged for integrating network-based platforms and pursuing interdisciplinary research related to mechanics, information, electronics, biotech, MEMS/NEMS (Micro Electro Mechanical Systems/Nano Electro Mechanical Systems), medical technology, and cloud computing. Automation is an inherently interdisciplinary field and we can say its interdisciplinary research falls into two broad approaches. The first approach identifies new ways to establish cross-functional teams and organizations to drive new interdisciplinary research areas while keeping the original organization intact. The second approach attempts to create a brand new organization which pursues interdisciplinary research.

\section{Lessons Learned from the History of Mechanics}

The two oldest logical sciences are mathematics and mechanics. Mechanics includes the physical fundamental elements for providing logical models such as time, mass, force, energy, heat and temperature. Archimedes developed many lessons and inventions in Mechanics such as the laws of levers and pulleys which allow for the movement of heavy objects using small forces, an example which is the Archimedean screw used to pull water out of the ground. As a testament to his genius, many of his innovations are still in use today. Looking at the historical background of Applied Mechanics, we can better understand why and how the field came into existence.

\section{History of Mechanics}

The study of Applied Mechanics originated with Archimedes (A.C. 287-212) the mathematician, physicist and engineer who developed principles related to buoyancy, levers, and compound pulleys. Other key 
figures in the development of the field include the artist, engineer, mathematician and anatomist Leonardo da Vinci (1452-1519), followed by Simon Stevin (1548-1620) a Flemish mathematician working in geometry, physics and trigonometry, and Galileo Galilei (1564-1642) who contributed greatly to astronomy and physics. Other well-known figures such as Isaac Beeckman (1570-1637), René Descartes (1596-1650), Christiaan Huygens (1629-1695), Isaac Newton (1643-1727), Gottfried Wilhelm Leibniz (1646-1716), Jakob Bernoulli (1655-1705), Leonhard Euler (1707-1783), Joseph-Louis Lagrange (1736-1813) have also made significant contributions to the advancement of science and engineering.

\section{Rational Mechanics}

Between 1950 to 1980, Rational Mechanics was established and strongly promoted by Clifford Truesdell at Johns Hopkins University along with his former student, Walter Noll. Rational Mechanics seeks to construct mathematical models to better understand continuous mechanical phenomena, and can be used to describe much of what is observed or inferred in the laboratory-based experimental settings $[5,6]$. Tuesdell suggested Rational Mechanics [5, 6] was defined by three significant features. Firstly, Rational Mechanics is a mathematical study of motions generated from specific forces as distinguished from statics, and examines the forces of a system in equilibrium. Secondly, the mathematical analysis used in Rational Mechanics represents the generation of trajectories of moving bodies as distinguished from geometry, which describes only static curves. Thirdly, the current formulation of Rational Mechanics is based on Newton's Principia Mathematica as opposed to practical mechanics which originates from classical and Hellenistic antiquity.

In the 1970s, trends in Rational Mechanics included open problems concerning Eulerian and Navier-Stokes fluids, and solutions to key problems of finite elastic deformation. Maxwell's kinetic theory of gases was shown to yield definite predictions about certain gas flows, rather than approximations based on nineteenth-century hydrodynamics. The ergodic theory of George David Birkhoff (1884-1944) and others was shown to provide a satisfactory basis for statistical mechanics of equilibrium, and serve as a partial response to the invention of various new materials. Moreover, the concept of oriented materials, for which the prototype is the Bernoulli-Euler-Kirchhoff theory of elastic rods, was extended to model various substances of current interest, especially liquid crystals. The concept of force was formalized through a set of axioms, much as points and lines are formalized in geometry and mass is formalized in measure theory. The second law of thermodynamics can generally be applied to deformable bodies undergoing processes of any kind. A general thermomechanics principle of materials possessing memory was constructed and the theory of wave propagation was developed and extended to thermomechanical materials with memory $[5,7]$.

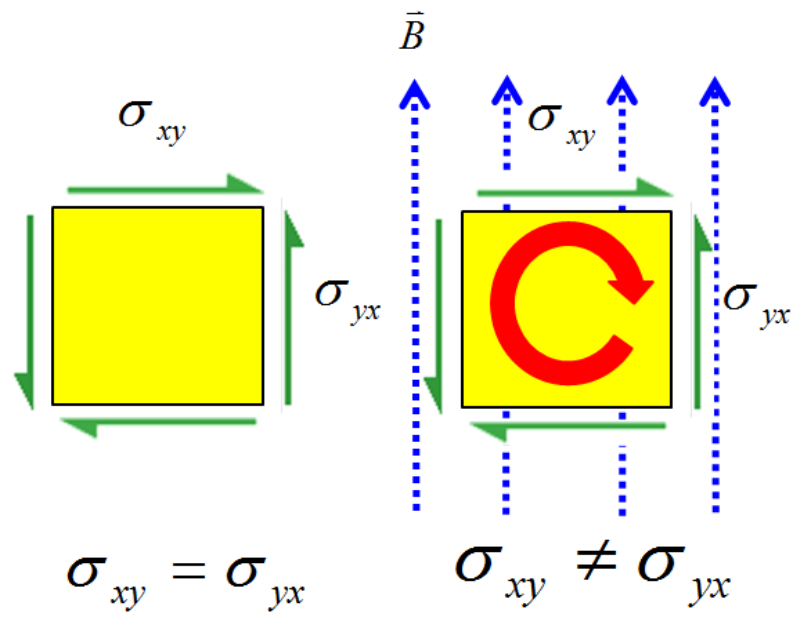

(a) No magnetic field present and the two marked shear stresses are identical due to equilibrium of forces

(b) Magnetic field present and the induced magnetic dipole will introduce extra stresses to achieve a new equilibrium state where $\sigma_{x y} \neq \sigma_{y x}$.

Figure 1. Fundamental difference of shear stresses for ferromagnetic materials with respect to the presence of an externally applied magnetic field.

The above-mentioned approach appears to provide an ideal approach of using mathematical models as a primary guideline for examining and exploring the physical world. However, a departure from this approach clearly emerged after more than 20 years of development. That is, the predictions of Applied Mathematics are not similar to the real physical world we live in as Applied Mathematics is based on perfect logic while the physical world is actually imperfect. For example, wave equations correspond to hyperbolic equations and heat equations correspond to parabolic equations. In mathematics, the difference between a hyperbolic equation and a parabolic equation is whether the wave speed approaches infinity or not. Nevertheless, in the physical world, neither wave speed approaches infinity. More importantly, historical experience has shown that this lack of perfection is a key driver of innovation and discovery. These findings have led to an improved understanding of the physical world purely through examining the Applied Mathematics model which branched off from mainstream thinking.

Nuclear of Fusion: A Turning Point for Creating an Independent Applied Mechanics Institute in Academia

Applied Mechanics emerged as an independent 
discipline with the formal establishment of the International Union of Theoretical and Applied Mechanics (IUTAM) in 1924. However, the establishment of Applied Mechanics as an independent research organization did not occur until the 1950s with the pursuit of nuclear fusion as a clean energy alternative. However, at the time, nuclear fusion research was conducted under unprecedented difficult conditions. To achieve the plasma state needed for nuclear fusion, temperatures of 30,000 degree $\mathrm{K}$ needed to be contained in a well-controlled manner. The only method known to properly contain such high-temperature plasma required the use of a strong magnetic field. It required an interaction between magnetic and elastic fields for ferromagnetic materials, which meant that a large structure to house the strong magnetic field was required. This requirement essentially involved coordination between physicists and civil engineers.

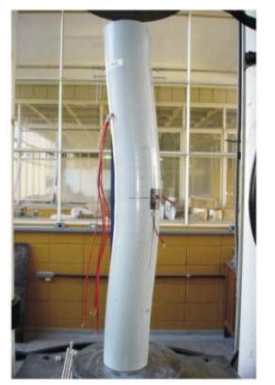

(a)

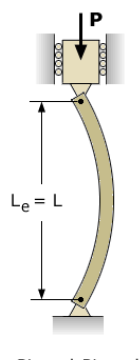

(c)

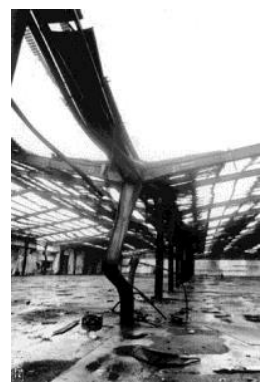

(b)

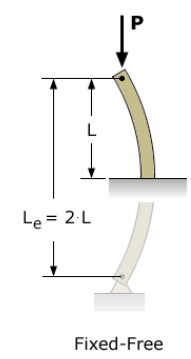

(d)
Figure 2. (a) Buckling of rod-type structure in a laboratory set-up, (b) photo of a real structural buckle, (c) analytical buckling model to analyze pinned-pinned ends, and (d) analytical buckling model to analyze fixed-free ends

More specifically, it was found that a buckle appeared far below the traditionally expected axial loading for steel structures located within a strong magnetic field. Buckling is a known mathematical instability leading to a failure mode not appearing in the direction of the load that the structure experiences (Fig. 1). Some structural buckles are shown in Fig. 2 [8]. The buckling phenomenon had not been encountered before in other disciplines. Moon [10] and Yeh [10] developed a magnetoelasticity theory to better understand this phenomenon. Even after many years of theoretical development, there still exists more than one generally accepted analytical formulation of using magnetoelasticity to examine the structural behavior of ferromagnetic materials under influence of a strong magnetic field.

\section{Applied Mechanics in Academia}

After the field of Applied Mechanics emerged as a separate discipline to handle issues related to nuclear fusion, it was established as a separate engineering department and even as an independent institute within Engineering schools at several renowned universities. The field of Applied Mechanics came to be viewed as an interdisciplinary field situated between Engineering and Science. Theoretical \& Applied Mechanics (T\&AM) departments were established at Cornell University, University of Illinois at Urbana-Champaign, Caltech and the University of Wisconsin-Madison. Based on these successful precedents, in 1983, National Taiwan University also set up its own Institute of Applied Mechanics (NTU-IAM) and began to accept graduate students the following year.

The National Taiwan University, Institute of Applied Mechanics (NTU-IAM) gradually established a reputation as a world-class institute, with its faculty working with academics from other disciplines around the world on a wide range of projects. At the outset, NTU-IAM research mainly focused on defense applications. However, over time, with defense budget cuts, the Institute shifted its focus to work on more emerging topics such as MEMS and NEMS-based projects. The Institute's innovative work on MEMS and NEMS brought it global recognition, and the results of its work have been emulated by other well-established academic disciplines such as Mechanical Engineering and Electrical Engineering. Currently, the predominant trend for institutes of Applied Mechanics around the world is its integration into other engineering departments or simply being phased out.

We discuss some of the underlying factors driving these changes in the field of Theoretical and Applied Mechanics and discuss the future for the field in an attempt to better understand the approach of other interdisciplinary research. The establishment and outcome of an interdisciplinary field such as Applied Mechanics provides good historical background for current or emerging interdisciplinary fields. The evolution of Applied Mechanics as a discipline shows that new ideas are driven by a specific demand, and successful ideas drive the growth of new research fields. 
Thus, newly established research fields can either move quickly to pursue new emerging fields or continue with the status quo. From past history, we can predict that the former will flourish while the latter will either be absorbed by other traditional engineering disciplines or become obsolete.

The development and evolution of Applied Mechanics (Fig. 3) suggests that advancing interdisciplinary research requires continuously scouting emerging fields. Gartner's 2012 Hype Cycle of emerging technologies (Fig. 4) [11] suggests that techniques such as 3D bio-printing, human augmentation, computer-brain interfaces, internet of things, etc., will most likely eventually become part of our lives and will be embedded in the discipline of Mechanics. Furthermore, these subjects are not found in traditional research fields as they require interdisciplinary knowledge. More specifically, these fields have characteristics similar to the driving forces which previously led to the establishment of Applied Mechanics, i.e., cross-interaction between engineering and basic sciences.

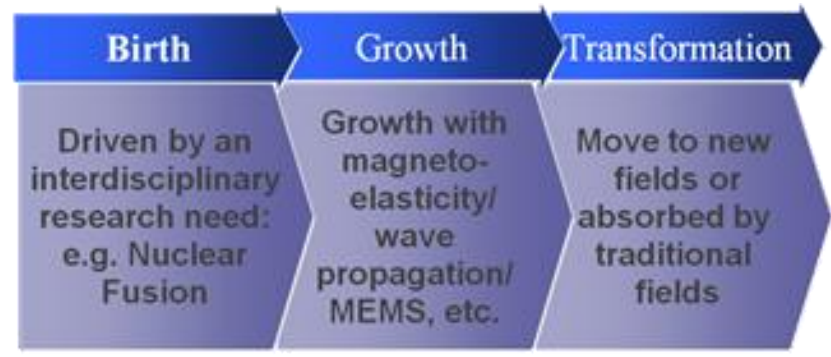

Figure 3. Development and change in Applied Mechanics

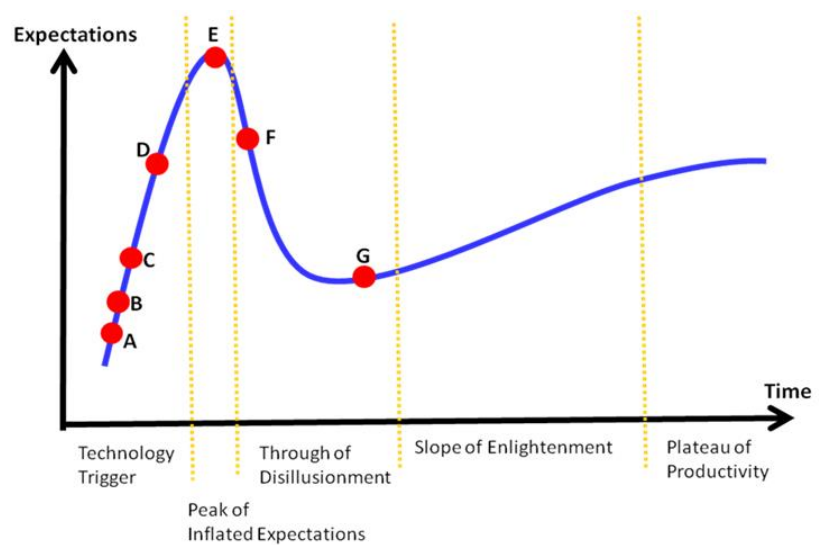

Figure 4. Excerpt of 2012 Hype Curve of emerging technologies by Gartner [11] (A: 3D Bio-Printing, B: Human Augmentation, C: Computer-Brain Interfaces, D: Internet of Things, E: Private Cloud Computing, F: Mesh Networks: Sensors, G: E-book Readers.)

\section{Lessons Learned from the History of Mechanics}

The two oldest logical sciences are mathematics and mechanics. Mechanics includes the physical fundamental elements for providing logical models such as time, mass, force, energy, heat and temperature. Archimedes developed many lessons and inventions in
Mechanics such as the laws of levers and pulleys which allow for the movement of heavy objects using small forces, the Archimedean screw to pull water out of the ground, etc. Many of these innovations are still in use today. Looking at the historical background of Applied Mechanics, we can better understand why and how the field came into existence.

\section{Reflections on Pursuing Nano-BioMEMS /NEMS, Smart Structures, and Application Platforms from an Applied Mechanics Perspective}

\section{Nano-optics \& Laser Micro-machining: Extraordinary Transmission Phenomenon}

As illustrated by our research group's work on Laser Micro-machining, the drive to move from fundamental advancement in basic science to engineering applications today demands considerable in-depth exploratory study both in basic sciences and engineering. The case study discussed below is used to illustrate the organizational and team efforts needed to integrate findings from basic sciences and engineering.

One of the fundamental advancements in laser micro-machining can be attributed to an extraordinary transmission phenomenon as discovered by Thomas Ebbesen. In 1998, Ebbesen et al. [13] published a paper discussing what he called an extraordinary optical transmission phenomenon. He found that a Maxima transmission efficiency can exceed unity when normalized to the area of holes, which is orders of magnitude greater than that predicted by standard aperture theory. He proposed that the unusual optical properties were due to the coupling of light with plasmons (Fig. 5(a)). This discovery opened a brand new direction to advance the technology of laser micro-machining.

In addition, the periodic texture created on the exiting side of a single aperture in a metal film creates a transmitted light which emerges from the aperture and appears as a beam with a small angular divergence (approx. $3 \mathrm{deg}$ ) and directionality that can be controlled. This finding led to the development of a novel method of creating a Bessel beam with a sub-micrometer spot size, and become a platform to create high-aspect-ratio microstructures. The findings of this extraordinary transmission and directional beaming provide the potential for the efficient fabrication of high-aspect-ratio sub-micrometer microstructures using laser micro-machining.

Roadmap of Laser Micro-machining within NTU Nano-BioMEMS Group

The small spot size of a Bessel beam makes it an 
ideal laser machining light source. Intrigued by this potential, the Nano-BioMEMS Group at National Taiwan University's Institute of Applied Mechanics set out to develop a laser micro-machining instrument for various industrial applications such as for 3DIC (3-dimensional integrated circuits), PC boards, etc. The newly created system was named a "Sub-wavelength Non-diffracting Beam Laser Writer". Figure 5 shows the research view and development flow of this system [14-17].

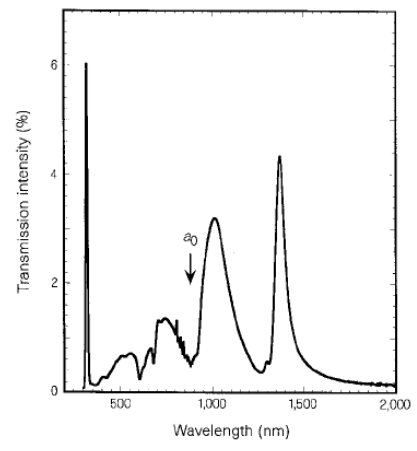

(a)

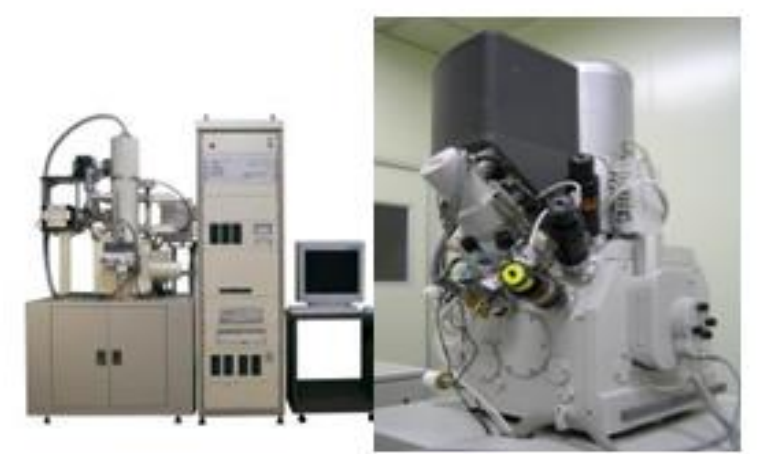

(c)

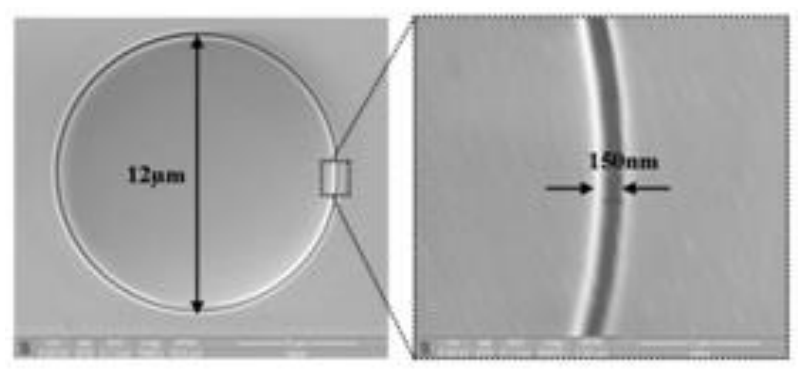

(d)

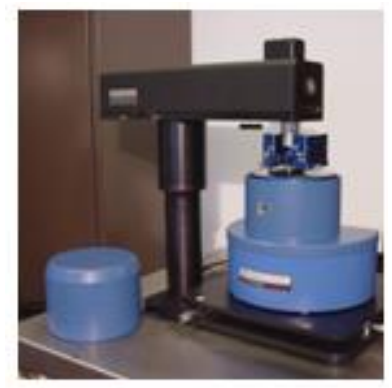

(e)

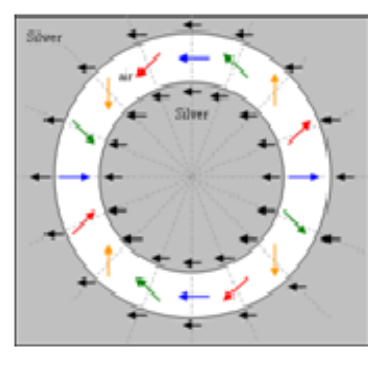

(b)

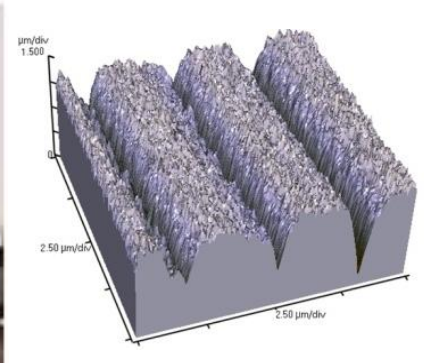

(f)

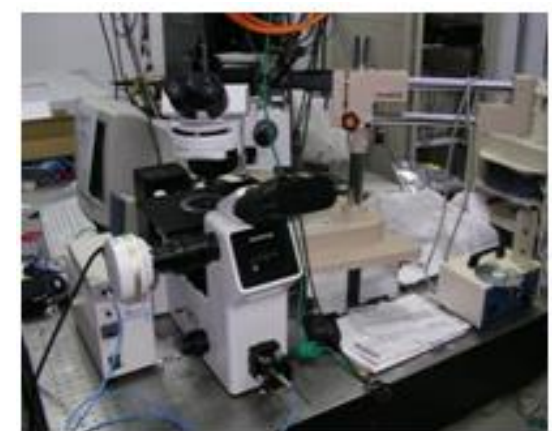

(g)

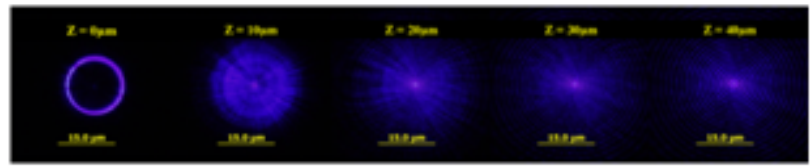

(h)

Figure 5. (a) Zero-order transmission spectrum of an Ag hole array, (b) Sub-wavelength Annular Apertures (SAA), (c) Focused ion beam (FIB) set-up, (d) SAA sample fabricated by FIB, (e) Atomic Force Microscope (AFM) for measurement use, (f) sample surface as measured by AFM, (g) modified microscope for emitted light beam characterization, and $(h)$ emitted light beam from SAA sample. [13-17]

Starting from an optimized Bessel beam, various nano/micro optical configurations were fabricated for laser micro-machining use. At the turn of the century, nano-optics was just starting to emerge and the development of techniques for simulation and fabrication processes was needed to further advance its progress. A sub-micrometer Annular Aperture (SAA) was developed from these efforts as the starting point (Fig. 5(b)). A focused ion beam (FIB) set-up (Fig. 5(c)) was adjusted and tested to fabricate the openings on a metal film sputtered on top of a glass substrate (Fig. 5(d)). A spectrometer, various microscopes and atomic force microscope (AFM) (Fig. 5(e)) were incorporated to verify and check the agreement of the simulation and experimental results. Fig. 5(f) shows the surface of the sample fabricated by the FIB. Figure $5(\mathrm{~g})$ shows the modified microscope with the integrated spectrometer. The emitted light beam measured by the lab-made microscope (Fig. 5(g)) is shown in Fig. 5(h).

Many versions of a laser micro-machining instrument were designed and built. The underlying physics that resulted from the emitted light beam of the silver SAA structure is shown in Fig. 6(a). Figure 6(b) shows a photo of the submicron-structures developed using the Bessel beam. It should be noted that many of the techniques, tools, and metrology instruments were not considered a part of traditional Applied Mechanics or Automation research. Our work to target this research discipline was indeed an interdisciplinary research effort. A completely different educational curriculum was also developed to transfer the knowledge generated as well 
as to quickly train new students from various backgrounds as capable researchers in the emerging field. Looking back at the history of Applied Mechanics, all these issues were previously addressed when nuclear fusion was the main research focus.

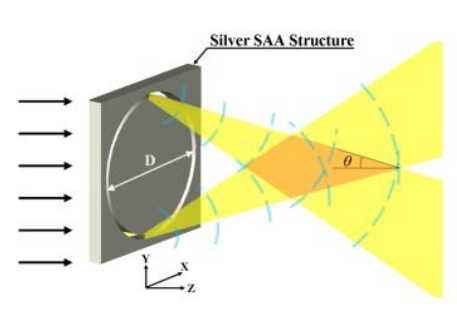

(a)

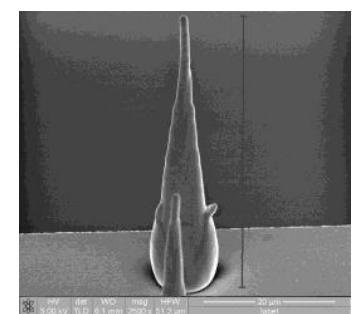

(b)
Figure 6 (a) Schematic of emitted light beam from silver SAA structure, and (b) photo of submicron-structures developed using the emitted Bessel beams from (a) [14-17].

\section{Serendipity}

The term "serendipity" was coined by Horace Walpole in a letter to his friend Sir Horace Mann in 1754 [18]. Walpole was impressed by a fairy tale he had read about the adventures of "The Three Princes of Serendip", who were always making discoveries by accident and finding "sagacity" of things for which they were not looking. Figure 7 illustrates the perceptual model of serendipity developed by numerous contributors over the years and can be described as follows:

1. (E-1) Be well prepared and be well trained in fundamental knowledge since "chance favors the prepared mind [19]."

2. (E) Bravely explore new fields/disciplines so as to encounter an unexpected event. Such unexpected encounters can also be achieved by active interaction via the internet and can be viewed as an example of "chance favoring the connected mind [20]".

3. $(E+1)$ Be keen to explore new opportunities when identified as "chance favors an open mind [21]."

4. $(E+2)$ Seize the moment to explore and to search for innovation.

5. $(E+3)$ Amplify the unexpected effects to recognize the potential of the unexpected event as discussed in $(E+2)$ which can maximize opportunity and the potential for a new discovery.

6. Continue to search for new opportunities and maximize potential and associated benefits.

The perceptual model of serendipity and the suggestion that chance favors the prepared/connected/ open mind clearly offers a guideline for training and for establishing innovation, even in our internet era.

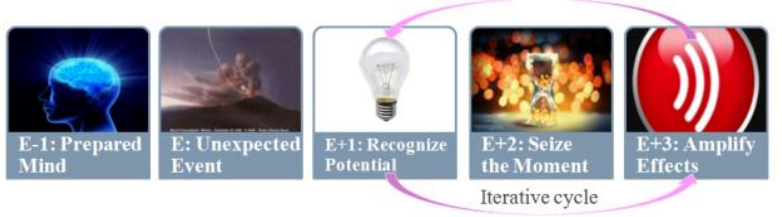

Figure 7. Perceptual model of serendipity.

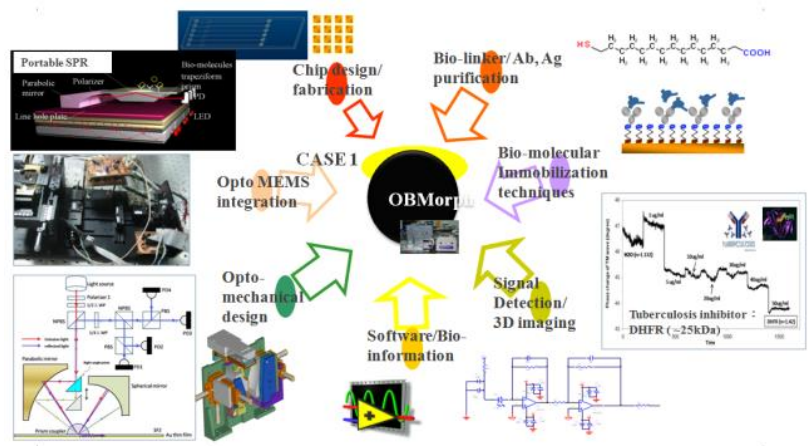

Figure 8. OBMorph and related technologies [22, 23].

Serendipity Factor in Interdisciplinary Research for Smart Structures, and Electret Application Platforms

For our purposes, one example of the above-mentioned perceptual model of serendipity is our newly developed "OBMorph", a multi-functional bio-oriented microscope which was used to successfully create protective gear and facemasks for use against the SARS (Severe Acute Respiratory Syndrome) virus during the SARS crisis [22, 23] in the early 2000s. The OBMorph work was also extended to create a paper speaker (FleXpeaker) [24] that won various awards. The OBMorph project (Fig. 8) was proposed and executed by the NTU Nano-bio MEMS Group at the Institute of Applied Mechanics. The original goal of the group was to develop a multi-functional microscope including a surface plasmon resonance (SPR) component, an ellipsometer, and an interferometer, for use in applications from biotech to health care. Improved accuracy required an ellipsometric sample surface of close to atomic flatness, and required the synthesis of new compounds along with new receptors. As the execution period of the project coincided with the SARS epidemic in Asia, many innovations developed during this time far exceeded their original targets in terms of research scope and achievements. The team also had an underlying belief that a world-class team must also assume social responsibility and focus on research that can benefit others. The interdisciplinary character of the research team and the team's dedication to pursuing socially-useful outcomes was later identified as the key serendipity factor which drove the successful research $[22,23]$.

The first SARS victim in Taiwan was identified on 
April $22^{\text {nd }}, 2003$ at National Taiwan University Hospital. At the time, doctors had to depend on face masks for protection, but high quality facemasks such as 3M's N95 were in short supply as the public began to purchase them in large quantities ( $E$ in Fig. 7). These masks were originally designed to filter dry dust, but featured an electret which could filter particles as small as 0.3 micrometers, thus allowing it to effectively filter the SARS virus. However, the electret was found to lose its effect once humidity from the user's breath caused the static charge contained within the electret to leak out. Once the static charge dissipated, the N95 face mask became just like a regular fabric facemask which offered no protection against the SARS virus. Our team then developed a new bio-linker compound which used a hydrogen bond to retain the biomolecules in an aqueous condition, which could then be sprayed on the embedded layers of the $\mathrm{N} 95$ face mask ( $E+1$ in Fig. 7). We found that the bio-linker bound the SARS virus biomolecules within the face mask. Our further research led us to create a derivative of the original bio-linker which could denature the SARS virus using a nanotechnology principle which caused the hydrophilic and hydrophobic properties to repel each other [26]. The newly developed chemical compound was coined the "NTU Anti-SARS No. 1 Compound" ( $E+2$ in Fig. 7). During the process of transferring this technology to industry, the NTU Nano-bio MEMS group worked with a facemask manufacturer to apply a corona charge onto the non-woven fabric sprayed with the anti-SARS compound. An electric field of more than 1500V was induced and, even with a typical decay, the non-woven fabric was found to retain a $1000 \mathrm{~V}$ electric field, offering a static potential more than twice that previously used in the fluoropolymer in 3M's N95 facemasks. This was surprising since the NTU Anti-SARS No. 1 Compound was actually nothing more than a lipid ( $E+1$ and $E+2$ in Fig. 7). In fact, it was a type of non-fluoropolymer electret which was a counterexample to many known successful electrets. The team also found that the electret could be used to develop an electret speaker (see Fig. 9) which provides good impedance with air and can provide a good medium for high frequency response. The paper speaker, called FleXpeaker, was jointly developed by the NTU Nano-BioMEMS Group and the Industrial Technology Research Institute (ITRI) [24]. A commercial application of the flexible electret speaker was displayed at the 2010 Taipei International Flora Exposition (Fig. 10). This product also won a Wall Street Journal Consumer Electronics Technology Innovation Award in 2009 [27]. The serendipity and roadmap of the research cases discussed are clearly depicted in Fig. 11.

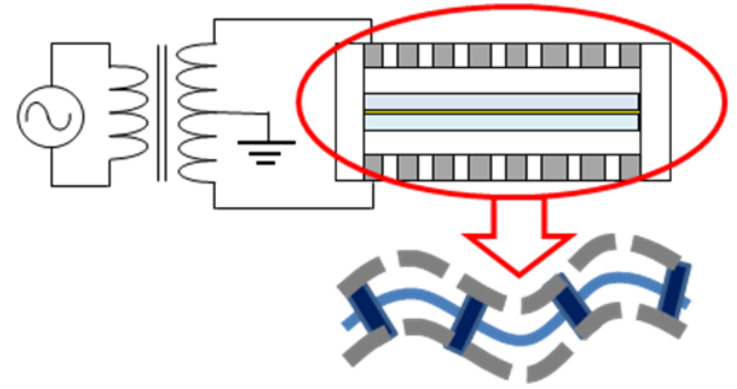

Figure 9. Structure of Electrets [24].

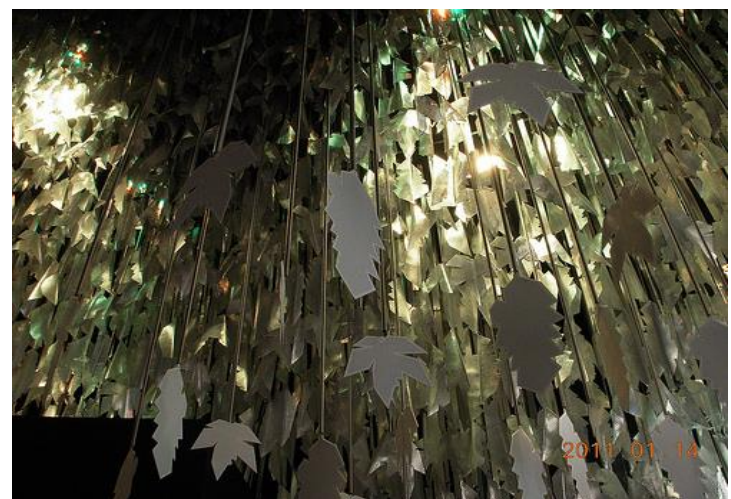

Figure 10 Flexible speakers at the 2010 Taipei International Flora Exposition.

\section{Conclusions}

Early requirements for nuclear fusion research led to the development of Applied Mechanics as a discipline. This paper discusses the drivers in the creation of various independent research institutes in Applied Mechanics, along with the role of serendipity on the development of an interdisciplinary field. An examination of the establishment and evolution of independent institutes centered on the discipline of Applied Mechanics suggests the need to continue to seek out new interdisciplinary research directions. Past history suggests that we create associated educational curriculum to promote the continued development of interdisciplinary research so as to facilitate successful research outcomes. University-industry collaborations have been shown to be an effective tool to come up with new ingredients in the search for new research directions.

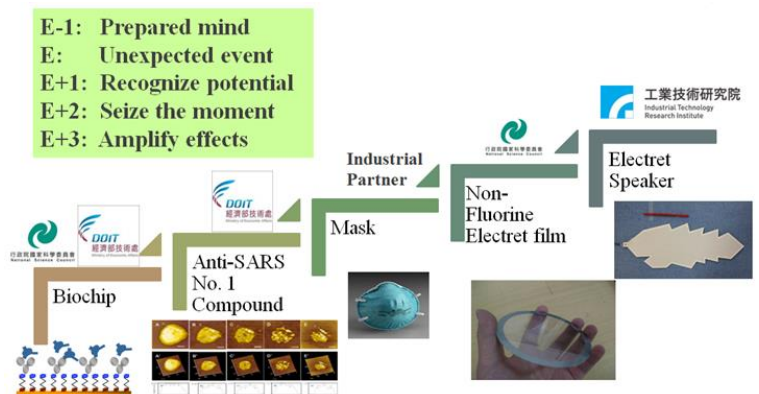

Figure 11. Serendipity and roadmap of NTU Nano-BioMEMS/NEMS Group projects 


\section{Acknowledgments}

This study was supported by Taiwan's National Science Council under Grants NSC 102-2221-E-002-188-MY3, NSC 100-2221-E-002-109-MY3, NSC 101-2627-E-002-003, NSC 102-2627-E-002-004, MOST 103-2627-E-002-004 and by Taiwan's Ministry of Economic Affairs under Grants MOEA 91-EC-17-A-05-S1-0017, MOEA 92-EC-17-A-05-S1-0017, MOEA 93-EC-17-A-05-S1-0017, MOEA 94-EC-17-A-05-S1-0017, MOEA 95-EC-17-A-05-S1-0017, and MOEA 96-EC-17-A-05-S1-0017.

\section{References}

[1] StateMaster-Encyclopedia: Wilhelm von Humboldt [Online].http://www.statemaster.com/encyclopedi a/Wilhelm-von-Humboldt.

[2] The history of Cavendish [Online]. http://www.phy.cam.ac.uk/history.

[3] K. Robinson, The importance of creativity [Online].http://life-engineering.com/do-public-sch ools-kill-creativity/.

[4] Automation Division of Ministry of Science and Technology (2010, March 19th). [Online]. http://www.etop.org.tw/ispui/program/automatio n/program.showProgramPage.Action?do=Introduct ion.

[5] C. A. Truesdell, A first course in rational continuum mechanics Vol. 71: Access Online via Elsevier, 1992.

[6] C. Truesdell, "History of classical mechanics," Naturwissenschaften, vol. 63, pp. 119-130, March 1 st 1976.

[7] C. C. Wang, and Truesdell, C, "Introduction to rational elasticity", Vol. 1, 1973.

[8] F. C. Moon, "Buckling of a superconducting ring in a toroidal magnetic field," Journal of Applied Mechanics, vol. 46, no. 1, pp. 151-155, 1979. doi: $10.1115 / 1.3424487$

[9] Y. H. Pao, "Applied Mechanics," in Applied Mechanics Annual Conference Speech, 1990.

[10] P. Yih-Hsing and Y. Chau-Shioung, "A linear theory for soft ferromagnetic elastic solids," International Journal of Engineering Science, vol. 11, no. 4, pp. 415-436, 1973. doi: 10.1016/0020-7225(73)90059-1

[11] Gartner's 2012 Hype Cycle for Emerging Technologies Identifies "Tipping Point" Technologies That Will Unlock Long-Awaited Technology Scenarios [Online]. http://www.haumu.com.tw/news/gartnerfabu201 1nianxinxingjishuchengshuduzhouqibaogao/10303 2553-2012Emerging-Technologies-Hype-Cycle-Aug ust-16-EMEA-3-pdf.pdf

[12] Problem Based Learning, wiki [Online].
http://en.wikipedia.org/wiki/Problem-based learni ng.

[13] T. W. Ebbesen, H. J. Lezec, H. F. Ghaemi, T. Thio, and P. A. Wolff, "Extraordinary optical transmission through sub-wavelength hole arrays," Nature, vol. 391, no. 6668, pp. 667-669, 1998.

doi: $\underline{10.1038 / 35570}$

[14] Y. Y. Yu, C. K. Chang, M. W. Lai, L. S. Huang, and C. K. Lee, "Ablation of silicon by focusing a femtosecond laser through a subwavelength annular aperture structure," 2010, vol. 7789, pp. 77890M-77890M-77896.

doi: $10.1117 / 12.859854$

[15] Y.-Y. Yu, D.-Z. Lin, L.-S. Huang, and C.-K. Lee, "Effect of subwavelength annular aperture diameter on the nondiffracting region of generated bessel beams," Optics Express, vol. 17, no. 4, pp. 2707-2713, 2009. doi: 10.1364/OE.17.002707

[16] D.-Z. Lin, C.-H. Chen, C.-K. Chang, T.-D. Cheng, C.-S. Yeh, and C.-K. Lee, "Subwavelength nondiffraction beam generated by a plasmonic lens," Applied Physics Letters, vol. 92, no. 23, pp. 233106-233106-233103, 2008.

doi: $10.1063 / 1.2943274$

[17] C.-K. Chang, D.-Z. Lin, C.-S. Yeh, C.-K. Lee, Y.-C. Chang, M.-W. Lin, J.-T. Yeh, and J.-M. Liu, "Experimental analysis of surface plasmon behavior in metallic circular slits," Applied Physics Letters, vol. 90, no. 6, pp. -, 2007. doi: $10.1063 / 1.2471966$

[18] Serendipity, wiki [Online] http://en.wikipedia.org/wiki/Serendipity

[19] Louis Pasteur, wiki [Online]. http://en.wikiquote.org/wiki/Louis Pasteur

[20] Steven Johnson, Where Good Ideas Come From: The Natural History of Innovation, Riverhead Trade, October 2010.

[21] An open mind leaves a chance for someone to drop a worthwhile thought in it, wiki [Online]. http://thinkexist.com/quotation/an open mind le aves a chance for someone to drop/185154.ht $\underline{\mathrm{ml}}$

[22] C. K. Lee, C. W. Lin, S. Lin, A. S. Y. Lee, J. Y. Wu, S. S. Lee, et al., "From an Integrated Biochip Detection System to a Defensive Weapon Against the SARS-CoV Virus: OBMorph," in MRS Proceedings Nanoengineered Assemblies and Advanced Micro/Nanosystems, Vol. 820, Paper No. 09.8, 2004 MRS Spring Meeting - Symposium On Advanced Microsystems-Integration with Nanotechnology and Biomaterials, San Francisco, CA, USA 2004. doi: 10.1557/PROC-820-09.8

[23] W.-L. Hsu, S.-S. Lee, and C.-K. Lee, "Ellipsometric surface plasmon resonance," Journal of Biomedical Optics, vol. 14, no. 2, pp. 024036-024036-024038, 2009. 
doi: $10.1117 / 1.3116713$

[24] K. Wen-Ching, T. Chien-Kai, L. Ing-Yih, W. Wen-Jong, L. Adam Shih-Yuan, and L. Chih-Kung, "Use of 2-(6-mercaptohexyl) malonic acid to adjust the morphology and electret properties of cyclic olefin copolymer and its application to flexible loudspeakers," Smart Materials and Structures, vol. 19, no. 5, p. 055007, 2010. Available: http://stacks.iop.org/0964-1726/19/i=5/a=055007

[25] NIOSH-Approved N95 Particulate Filtering Facepiece Respirator [Online]. http://www.cdc.gov/niosh/npptl/topics/respirators /disp_part/n95list1.html

[26] S. Lin, C.-K. Lee, S.-Y. Lee, C.-L. Kao, C.-W. Lin, A.-B. Wang, S.-M. Hsu, and L.-S. Huang, "Surface ultrastructure of sars coronavirus revealed by atomic force microscopy," Cellular Microbiology, vol. 7, no. 12, pp. 1763-1770, 2005 . doi: 10.1111/i.1462-5822.2005.00593.x

[27] The Wall Street Journal 2009 Technology Innovation Awards [Online]. http://online.wsj.com/news/articles/SB100014240 $\underline{52970203440104574399714096167656 .}$ 